\title{
CRP Binding Kinetics Enhancement Using Local Narrowing into a Bent Channel: Finite Element Analysis
}

\author{
Mohamed Bahri 1,2,3*, Imen Dermoul ${ }^{4}$, Marshet Getaye ${ }^{3}$, Mohamed Amin Elaguech ${ }^{1,2,3}$, \\ Khouloud Djebbi 1,2,3, Mounir Ben Ali 5,6, Chaker Tlili1,2, Deqiang Wang1,2,3* \\ ${ }^{1}$ Chongqing School, University of Chinese Academy of Sciences, Chongqing, China \\ ${ }^{2}$ Chongqing Institute of Green and Intelligent Technology, Chinese Academy of Sciences, Chongqing, China \\ ${ }^{3}$ University of Chinese Academy of Sciences, Beijing, China \\ ${ }^{4}$ Preparatory Institute for Engineering of Nabeul IPEIN, University of Carthage, Campus Universitaire, Merazka, Tunisia \\ ${ }^{5}$ NANOMISENE Lab, LR16CRMN01, Centre for Research on Microelectronics and Nanotechnology of Sousse, Technopole of \\ Sousse, Sousse, Tunisia \\ ${ }^{6}$ University of Sousse, Higher Institute of Applied Sciences and Technology of Sousse, GREENS-ISSAT, Cité Ettafala, \\ Sousse, Tunisia \\ Email: ^bahrimohamed@cigit.ac.cn, ^Dqwang@cigit.ac.cn
}

How to cite this paper: Bahri, M., Dermoul, I., Getaye, M., Elaguech, M.A., Djebbi, K., Ali, M.B., Tlili, C. and Wang, D.Q. (2022) CRP Binding Kinetics Enhancement Using Local Narrowing into a Bent Channel: Finite Element Analysis. Engineering, 14, 62-75. https://doi.org/10.4236/eng.2022.141006

Received: December 14, 2021

Accepted: January 21, 2022

Published: January 24, 2022

Copyright $\odot 2022$ by author(s) and Scientific Research Publishing Inc. This work is licensed under the Creative Commons Attribution International License (CC BY 4.0).

http://creativecommons.org/licenses/by/4.0/

\section{(c) (i) Open Access}

\begin{abstract}
Binding kinetics enhancement of a microfluidic biosensor into a micro-channel through the application of a supplementary mechanism has received tremendous attention because of the obtained significant enhancement factor. However, biosensor's performance enhancement using only simple channel engineering is still rarely realized. Herein, we present a novel design of a complex reactive protein (CRP) biosensor into a U-shaped channel with a sensitive membrane located in the middle of the bent zone. Various critical factors affecting the equilibrium binding time are numerically investigated. The turn geometry is then optimized when the arc length along the inner and outer radii is almost the same, which leads to locally minimizing the channel height overhead the reaction surface and improves the analyte transport towards the sensing area. The numerical studies reveal that applying a local narrowing above the reaction surface can notably enhance the trapping and the surface formation of complex antibody-antigen, thus upgrading the biosensor performance. This work puts a significant advance towards microfluidic channel engineering and the exploration of micro-flow injection experimental studies.
\end{abstract}

\section{Keywords}

Finite Elements Method, Binding Reaction, CRP Biosensor, Microfluidic 
System, Microchannel Turn, Numerical Simulation

\section{Introduction}

Over the past decades, the U-shape tube has been widely used in measurement [1] [2]. One of the oldest measurements is pressure measurement, where the free end of the tube is reported with the atmosphere, and the pressure measurement is brought to another end [3]. Earlier studies have shown the application of a $\mathrm{U}$-shaped channel to measure some physical quantities, like concentration [4], temperature [5], density [6], and frequency [1]. Nowadays, after many performance investigations, the U-shape channel has been successfully implicated into various applications, including optical fiber in chemical probes such as glucose [7], pH levels [8], ethanol [9], salt [10], and toluidine blue [11]. Moreover, the U-shape micro-nanochannels can generate a locally amplified electric field and significant flow disturbance, uplifting its applicability in microfluidic mixers, electro-kinetic pumps, and cell lysis processes [12]. Various researchers have recently demonstrated the $U$ shape's utility in the design of biosensors for biomarker detection. For example, Wen et al. developed a U-shaped fiber biosensor using the lamping process for MicroRNA detection [13]. The developed biosensor showed high selectivity and an impressive limit of detection $(0.5 \mathrm{nM})$. A similar channel shape has been used for label-free detection of $E$. coli in which the designed biosensor exhibits a good sensitivity in solution measurements [14]. This shape also showed enhanced outcomes with a 10 -fold improvement in the absorbance sensitivity compared to the straight probes [15]. It is noticeable that numerous design and fabrication aspects related to the channel characteristics have been investigated, going from the repercussion of channel configuration geometry [16] to the amendment of the bent region [17]. Accordingly, several studies have treated the turn broadening and how channels could be designed to enhance the sensor performances. Griffiths and Nilson [18] studied the turning effect on the growth of the band variance of neutral species induced by electroosmotic transport. In another study, Paegel et al. [19] put forward the local narrowing of an electrophoresis duct width upstream of a bent and then widening it again after the bent zone. The current gold standard for such measurements relies on the straight duct shape with a constant area, as detailed in our previous work [20]. Therefore, there is a growing interest in developing various approaches to improve mass transport in microfluidic devices that are mainly based on a supplementary enhancement mechanism. For instance, Selmi et al. [21] suggested a microfluidic immuno-sensor, where an additional confinement flow was joined perpendicularly to the sample flow. Thus, the rise in the makeup flow allowed the analyte to be suppressed into a thin layer bordering the biosensor. In another work, Sigurdson et al. [22] proposed the application of the electrokinetically driven $\mathrm{AC}$ technique to ameliorate the response of the developed 
immuno-sensors, which greatly upgraded the antigen achievement to the immobilized ligands region. While, others have reported the use of magnetic field [23], obstacle above the biosensor [24], and electrothermal effect [25] to improve the target analyte achievement. Nevertheless, in some practical cases, controlling the channel geometry at a small scale via a supplementary enhancement system might be challenging and expensive due to the high fabrication costs.

Recently, there exists a great deal of exploring the pivotal role of chronic inflammation in cancer development [26]. The C-reactive protein (CRP) was considered as one of the most commonly studied chronic inflammation biomarkers [27], and multiple prospective studies revealed that patients with diverse types of cancer including lung cancer have moderately elevated circulating levels of CRP [28]. Therefore, there is an ultimate need for the development of a rapid and highly sensitive biosensor for CRP detection, where the existence of an enhancement system can put a significant stepping stone for upgrading the biosensor performance.

The above viewpoints have inspired the present work, where we employed the finite element method to simulate the CRP binding reaction through a U-shaped microchannel. Various crucial factors that affect the equilibrium binding time are discussed, such as the inlet flow velocity, the bulk analyte concentration, and the microchannel's equivalent height. An enhancement factor of about 1.20 was obtained using simple local narrowing of the microchannel above the biosensor. We find that upgrading the binding reaction without any supplementary enhancement mechanism via only simple channel engineering could have a significant role to fabricate microfluidic gadgets.

\section{Theoretical Model}

The recognition between an analyte carried by a fluid with a concentration $[A]$ (CRP), and immobilized ligands $[B]$ (anti-CRP), is occurring at a $2 \mathrm{D}$ membrane with reaction kinetics leading to form the complex CRP/anti-CRP. The surface reaction can be depicted in a two-step process as follows:

1) $[A]_{\text {Bulk }} \rightleftarrows[A]_{\text {Surface }}$

2) $[A]_{\text {Surface }}+[B] \underset{K_{\text {off }}}{\stackrel{K_{\text {on }}}{\rightleftarrows}}[A B]$

In this case, $[A]_{\text {Bulk }}$ represents the analyte's bulk concentration, and $[A]_{\text {Surface }}$ is the reaction surface's analyte concentration. $[B]$ refers to the surface-immobilized ligand concentration, and $[A B]$ represents the complex analyte-ligand surface concentration. $K_{\text {on }}$ and $K_{\text {off }}$ are the association and dissociation rate constant, respectively. The first process (1) is called mass-transport, controlled by diffusion mechanism and governing the analyte transport from the bulk toward the reaction surface. The second process (2) is the binding reaction; a chemical reaction that describes the surface complex's formation. In this work, we assume that the developed immunoassay mixes the concentration of biological analyte human CRP in a neutral buffer solution that can be the phosphate buffer saline, which presents physical characteristics comparable to the water [29]. Hence, was 
utilized as a carrier fluid.

1) Microchip design

The flow is static under the condition of a non-slip flow for the wall condition, where the analyte is transported toward the chemical reaction surface by convection and diffusion. This whole phenomenon involves an expansion of a diffusion boundary layer, which depends on the ratio of the surface reaction rate, and the bordering biosensor's flow velocity. The simulated device properties are shown in Figure 1.

The channel width is assumed to be $100 \mu \mathrm{m}$. The straight section's length $(L)$ of the microchannel is $1 \mathrm{~mm}$, and the outer radius $\left(r_{0}\right.$ for the curved microchannel is $150 \mu \mathrm{m}$. The fluid containing biological analyte (CRP) concentrations is injected at $x=1000 \mu \mathrm{m}$, flows from the left up to the left down boundaries along the $\mathrm{x}$-axis, crosses the turn, and retraces the path with flowing along the $x$-axis again to attain the outlet boundary. For all the simulations, the biosensor length is sustained correlative to the reaction surface which is $100 \mu \mathrm{m}$. The biosensor is centered in the middle of the turn at $140 \mu \mathrm{m}$, and $0 \mu \mathrm{m}$ at the $\mathrm{x}$-axis and $y$-axis, respectively. The problem is then unriddled based on the Fick second law coupled with the Navier-Stokes equations, and the first order Langmuir adsorption model for the reaction rate between the analyte and the ligand was employed.

\section{2) Mathematical formulations}

The developed approach is based on the finite element method [30] for a microfluidic biosensor in a two-dimensional microchannel, and thus, the surface concentration of complex analyte-ligand versus time was calculated. The mathematical model was used to simulate the binding reaction between a solution A (CRP) transported by a fluid to reach a sensitive boundary where a ligand $\mathrm{B}$ of (anti-CRP) is immobilized. A finite element platform (Comsol Multiphysics, COMSOL, Sweden v5.2a) [31] was utilized to compute a $2 \mathrm{D}$ simulation of momentum along with mass transport into the micro-scaled channel and overhead the biosensor surface.

\section{a) Navies-Stokes equations}

In this work, the fluid is assumed to be Newtonian and incompressible, with a laminar flow, isotherm, and steady-state. Hence the governing equations with dimensionless quantities can be described as the following equations [32] [33];

$$
\frac{\partial u}{\partial x}+\frac{\partial v}{\partial y}=0
$$

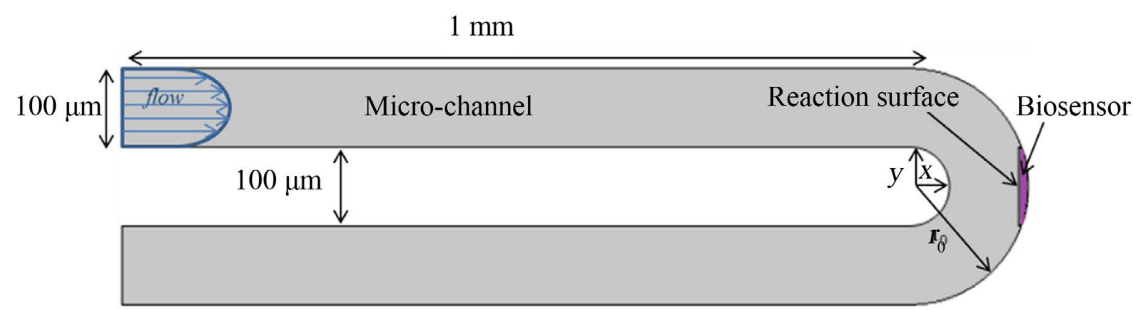

Figure 1. The 2D modeled structure. 


$$
\begin{aligned}
& \rho \frac{\partial u}{\partial t}+\rho\left(u \frac{\partial u}{\partial x}+v \frac{\partial u}{\partial y}\right)-\eta \nabla^{2} u+\frac{\partial p}{\partial x}=0 \\
& \rho \frac{\partial v}{\partial t}+\rho\left(u \frac{\partial v}{\partial x}+v \frac{\partial v}{\partial y}\right)-\eta \nabla^{2} v+\frac{\partial p}{\partial y}=0
\end{aligned}
$$

where $u$ and $v$ are the $x$ and $y$ velocity components, respectively, $\rho$ and $\eta$ are the fluid's density and dynamic viscosity, respectively, and $p$ is the pressure. Both $\rho$ and $\eta$ are constant and independent from the temperature or concentration. The flow field module is modeled via the Navier-Stokes equations associated with the initial and boundary conditions.

\section{b) Fick second law equation}

The analyte transport to land on the sensitive surface is depicted by the following equation [34]:

$$
\frac{\partial[A]}{\partial t}+u \frac{\partial[A]}{\partial x}+v \frac{\partial[A]}{\partial y}=D\left(\frac{\partial^{2}[A]}{\partial x^{2}}+\frac{\partial^{2}[A]}{\partial y^{2}}\right)
$$

where $D$ is the analyte diffusion coefficient and for human CRP its value is equal to $2.175 \times 10^{-11} \mathrm{~m}^{2} / \mathrm{s}$.

\section{c) Binding reaction}

The chemical kinetics equation is simulated through the first order Langmuir adsorption model [35] [36] assuming that the complex CRP/anti-CRP [ $A B]$ was achieved on the sensitive surface is bounded without diffusion and is raised as a function of time corresponding to the reaction rate equation as the follows:

$$
\frac{\partial[A B]}{\partial t}=K_{\text {on }}[A]_{\text {Surface }}\left\{\left[B_{0}\right]-[A B]\right\}-K_{\text {off }}[A B]
$$

where $[A]_{\text {Surface }}$ is the reaction surface analyte concentration, and $\left[B_{0}\right]$ is the immobilized ligand's concentration which is assumed to be $1.4 \times 10^{-8} \mathrm{~mol} / \mathrm{m}^{2} . K_{\text {on }}$ and $K_{\text {off }}$ are the association and dissociation rate constants, respectively, corresponding to $1 \times 10^{4} \mathrm{~m}^{3} / \mathrm{mol} \cdot \mathrm{s}$ and $2.6 \times 10^{-2} \mathrm{~s}^{-1}$ for the CRP-anti-CRP binding interactions.

\section{3) Numerical method and validation}

In this theoretical study, the stationary simulation was performed with constant pressure boundary conditions by setting $0 \mathrm{~Pa}$ at the channel's inlet and outlet. Additionally, a non-slip boundary condition was applied at the microchannel's walls. The flow is laminar with a low Reynolds number [37], isotherm, and steady. Whereas the fluid is initially supposed to be at rest. The initial concentrations of the complex CRP/antiCRP $[A B]$ and the bulk analyte concentration $[A]$ were set zero as initial conditions. In our case, the fluid was initially at rest, and the parabolic profile's average velocity was fixed at $u=10^{4} \mathrm{~m} / \mathrm{s}$ at the inlet boundary. The fluid parameters were supposed to be similar to the room temperature distilled water parameters $\left(\eta=1 \mathrm{mPa} \cdot \mathrm{s}, \rho=1000 \mathrm{~kg} \cdot \mathrm{m}^{-3}\right)$. And the [ $B_{0}$ ] was fixed at $1.4 \times 10^{-8} \mathrm{~mol} / \mathrm{m}^{2}$, and $K_{\text {on }}$ and $K_{\text {off }}$ were set at $1 \times 10^{4} \mathrm{~m}^{3} / \mathrm{mol} \cdot \mathrm{s}$ and $2.6 \times 10^{-2} \mathrm{~s}^{-1}$, respectively [20] [32].

The previously mentioned governing equations' solution using initial and 
boundary conditions applied in 2D has no analytical solution. Thus, Comsol software simulation offers the possibility to couple the laminar flow with the transport of diluted species physics module. To discretize the designed 2D domains, an unstructured triangular mesh was utilized; somehow, the neighboring area of the biosensor is more fine-grained than that of the bulk for a higher resolution [38], as displayed in Figure 2(a). Several mesh grids were applied to ensure the convergence and mesh independent of the numerical outcomes, as presented in Figure 2(b). Hence, no significant variation was found between the curves computed with 83070 and 56966 grids elements. Similar numerical convergence was found and explained as well [38]. Unless specified, all the computational studies were achieved with a total element number of 56,966.

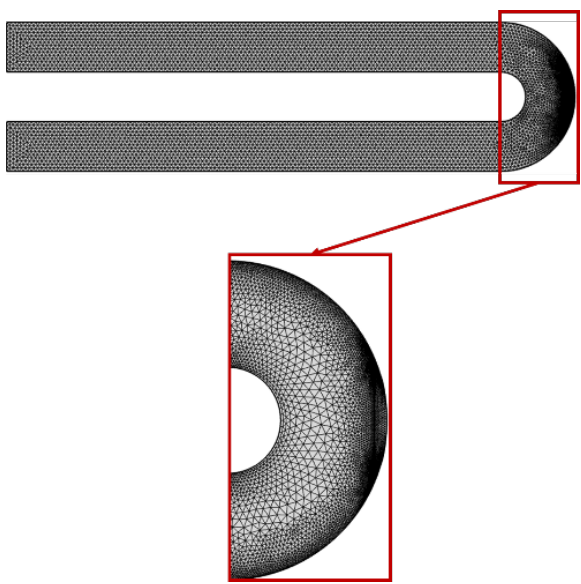

(a)

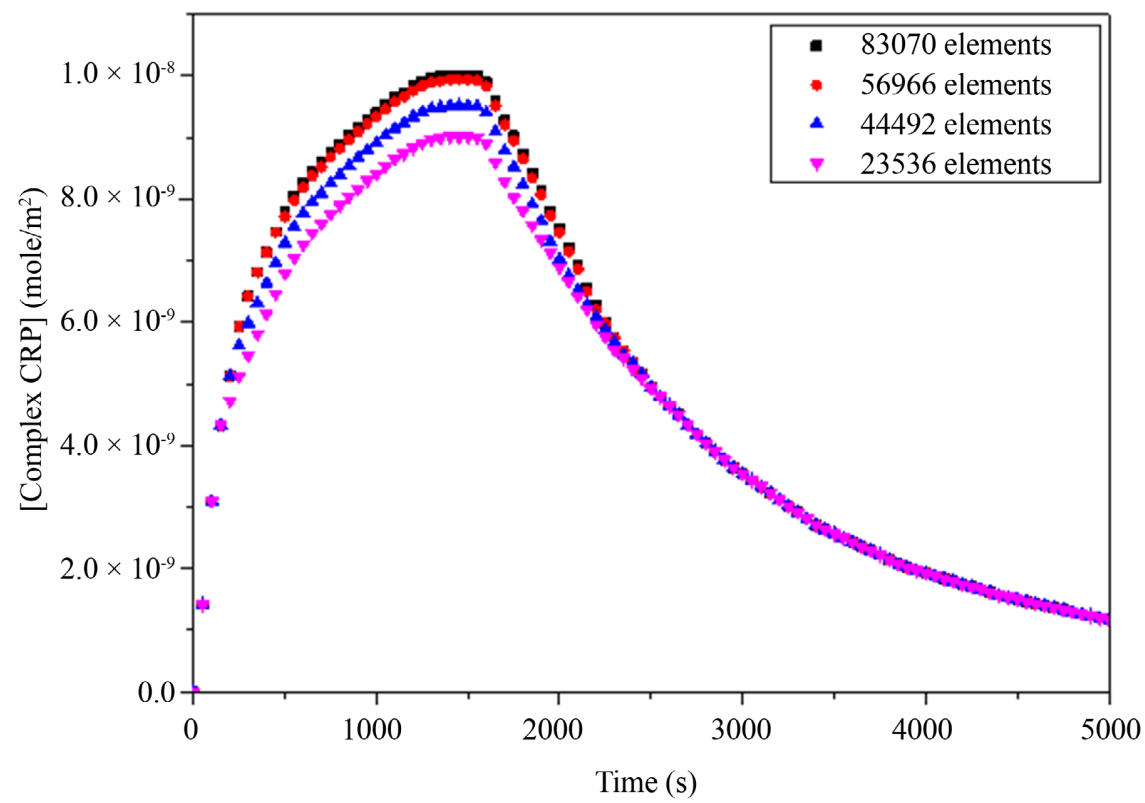

(b)

Figure 2. (a) The 2D triangular elements mesh geometry, (b) CRP complex concentration evolution in the function of time for different elements mesh number at a constant bulk concentration of $6.4 \mathrm{nM}$ and inlet flow velocity $U=100 \mu \mathrm{m} / \mathrm{s}$. 


\section{4) Binding kinetics of protein CRP and inlet flow velocity}

The surface complex concentration for the CRP versus different analyte concentrations, namely, 64, 12, 6.4, 1.2, and $0.64 \mathrm{nM}$, is depicted in Figure 3(a). The bulk analyte concentration rise leads to an increase of the trapped biomolecule concentration on the biosensor surface. The theoretical explanation behind the increase of the complex concentration $[A B]$ versus the analyte concentration $[A]$ is related to the Equation (7). Equation (7) can be obtained from Equation (5) through the equilibrium association state $\left(\frac{\partial[A B]}{\partial t}=0\right)$ as shown in Equation (6) $[32]$

$$
K_{\text {on }}[A]_{\text {Surface }}\left\{\left[B_{0}\right]-[A B]\right\}-K_{\text {off }}[A B]=0
$$

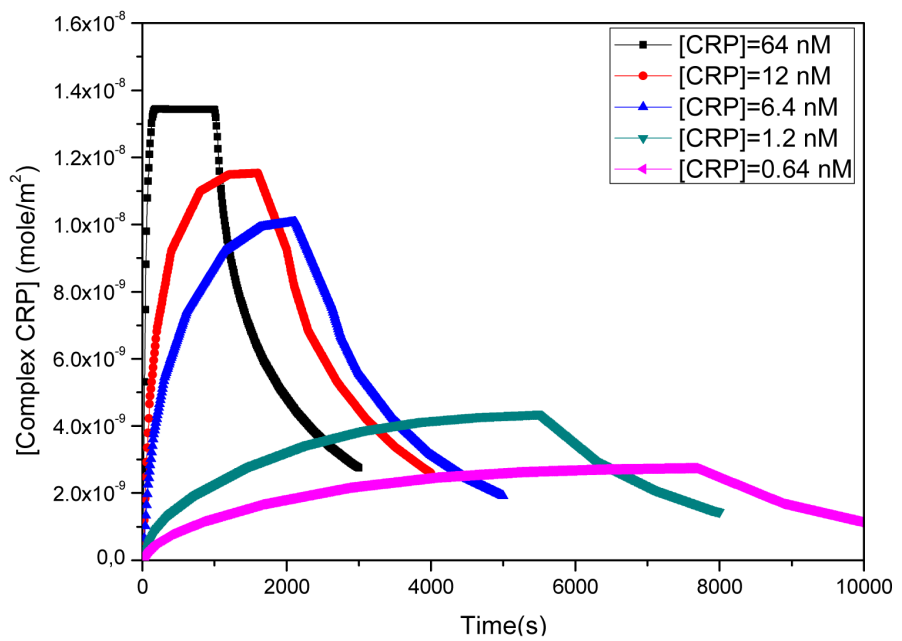

(a)

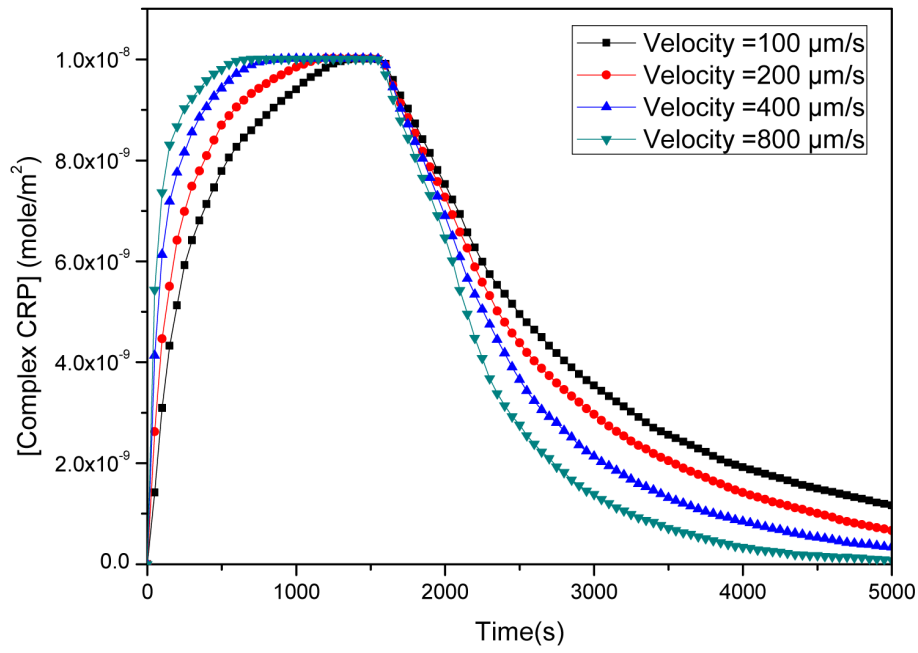

(b)

Figure 3. (a) The temporal evolution of the CRP complex concentration at the surface reaction for various bulk concentrations of CRP at a stable inlet flow velocity $U=100$ $\mu \mathrm{m} / \mathrm{s}$; (b) The effect of inlet flow velocity on the complex CRP's temporal evolution at a stable bulk concentration of $6.4 \mathrm{nM}$. 


$$
[A B]=\frac{K_{\text {off }}[A B] B_{0}}{K_{\text {on }}[A]+K_{\text {off }}}=\frac{[A] B_{0}}{[A]+K_{D}}
$$

where $K_{D}=\frac{K_{\text {off }}}{K_{\text {on }}}$ is the dissociation equilibrium constant. Additionally, the rise of the inlet flow velocity is effectively downsizing the diffusion boundary thickness, which notably enhances the rates of association and dissociation phases of the CRP/antiCRP binding reaction, as shown in Figure 3(b). The same explanation has been well detailed by Yang and co-workers, who pointed out the different parameters affecting the CRP binding reaction through a straight microfluidic duct [32]. The same numerical results are here used as a reference to validate the obtained data, which is matched well in terms of trapped complex concentration on the biosensor surface. Hence, association and dissociation rate, and time dependence are here unmatched due to the channel size and geometry difference.

\section{Results and Discussion}

\section{1) Diffusion boundary layer}

Due to its small diffusion coefficient, the protein as a large molecule is limited by the binding reaction's mass transport coefficient of the complex antigen-antibody structure [39]. Thus, this mass transport coefficient $\left(K_{m}\right)$ is defined by the flow velocity and the diffusion coefficient. $K_{m}$ can be defined from the Levich expression [40] as follows:

$$
K_{m}=0.98 * D^{\frac{2}{3}}\left(\frac{U}{L * H}\right)^{\frac{1}{3}}
$$

where $D$ is the diffusion coefficient, and $U$ is the inlet flow average, $L$ and $H$ are the position of the sensitive surface and the channel height, respectively. Consequently, the diffusion boundary layer can be given as a function of the diffusion and the mass transport coefficients as follows [41]:

$$
d_{\text {diff }}=\frac{D}{K_{m}}
$$

The diffusion boundary layer development will go up along the reaction boundary in the same flow direction. Thus, systematic mass transport is characterized by a lower thickness of the diffusion layer. Figure 4 shows the development of the diffusion boundary layer on the biosensor surface after different computational times. Accordingly, mass transport is occurring alongside the reaction surface following the same fluid path. Besides, during the association phase, the consumption of $[A]_{\text {Surface }}$ is briefer on the biosensor surface when compared with its consumption in the bulk. Consequently, a thin diffusion layer of the analyte above the biosensor is constructed explained by local consumption of the analyte concentration. Contrary to the association phase, a relatively higher analyte concentration covering the reaction surface than that of the bulk 


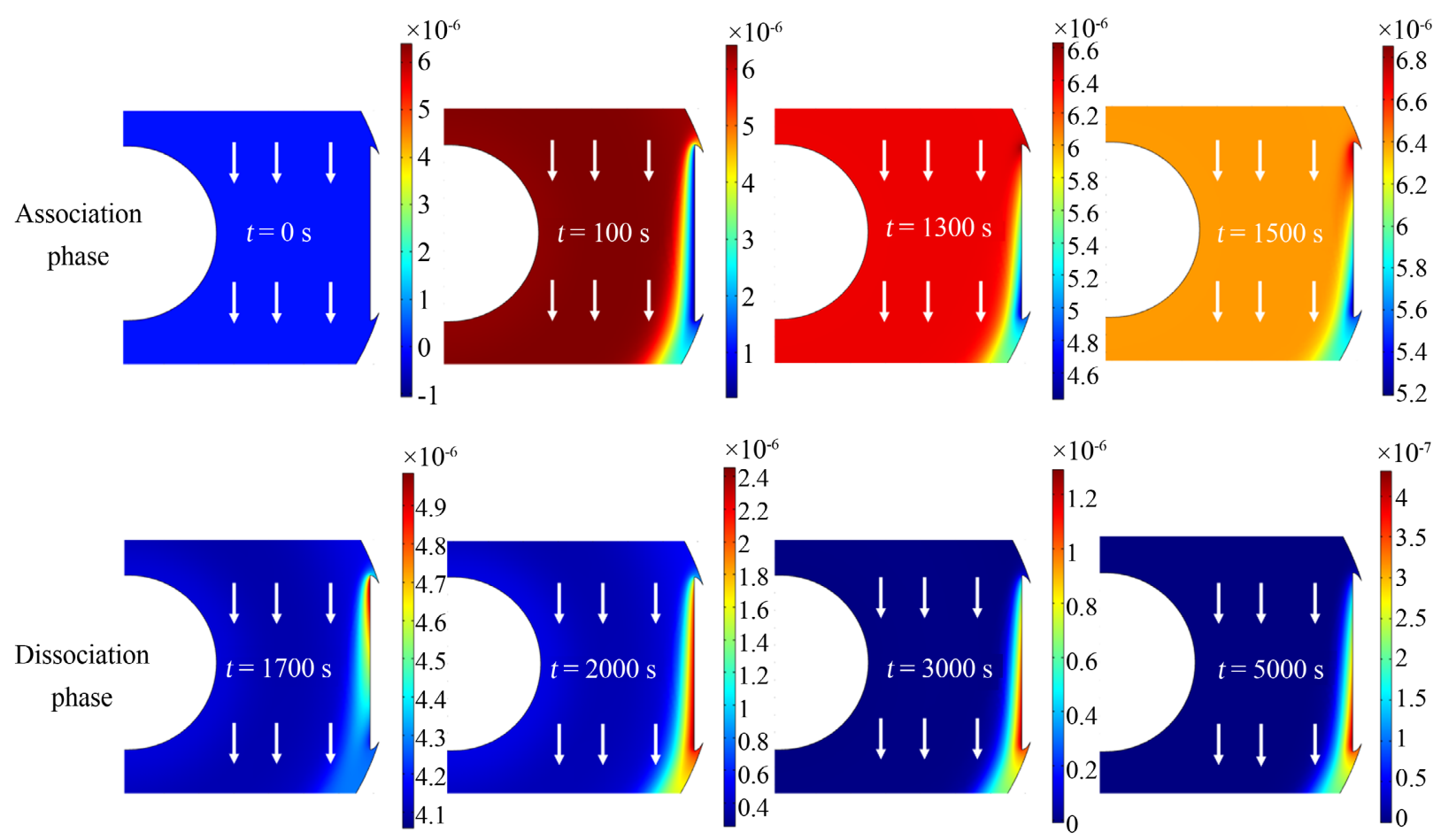

Figure 4. Development of diffusion boundary layer in association and dissociation phases at a CRP bulk concentration of $6.4 \mathrm{nM}$ and a constant average inlet flow velocity $U=100 \mu \mathrm{m} / \mathrm{s}$ at different times $t$.

can be clearly noticed during the dissociation phase, where no more analyte flows in the system [32].

2) Local narrowing method (reduction of the microchannel equivalent height)

As mentioned in the previous section, the relation combining the reaction channel height and the coefficient mass transport is resumed in the Levich Equation (8). Whence, decreasing the channel height $(H)$ can notably downscale the diffusion coefficient $(D)$ and promotes the mass transport coefficient $\left(K_{m}\right)$. In this subsection, the channel height overhead the reaction surface is varied depending on $\left(R_{0}\right)$ and $\left(R_{1}\right)$ as two specific narrowing dimensions presented in Figure 5(a) and detailed in Table 1.

Figure 5(b) displays the complex CRP concentration evolution versus time for different channel heights $\left(R_{1}\right)$. Here, it is worth mentioning that every computation of different microchannel heights was achieved at a constant bulk analyte concentration and an average flow velocity of $6.4 \mathrm{nM}$ and $100 \mu \mathrm{m} / \mathrm{s}$, respectively. Table 1 compiles the binding reaction initial slope of the association and dissociation phases. Note that by minimizing the channel height that covers the sensitive membrane from a drop case $(100 \mu \mathrm{m})$ to $40 \mu \mathrm{m}$, the binding reaction can be greatly enhanced, recording larger initial slopes from $1.76 \times 10^{-11}$ to $2.1 \times$ $10^{-11}$ for the association phase and from $-5.67 \times 10^{-12}$ to $-6.81 \times 10^{-12}$ for the dissociation phase. Such local narrowing leads to enhancement factors of 1.19 and 1.20 for the association and the dissociation phase, respectively. A larger slope of the CRP binding was found at a lower equivalent height. This can be 


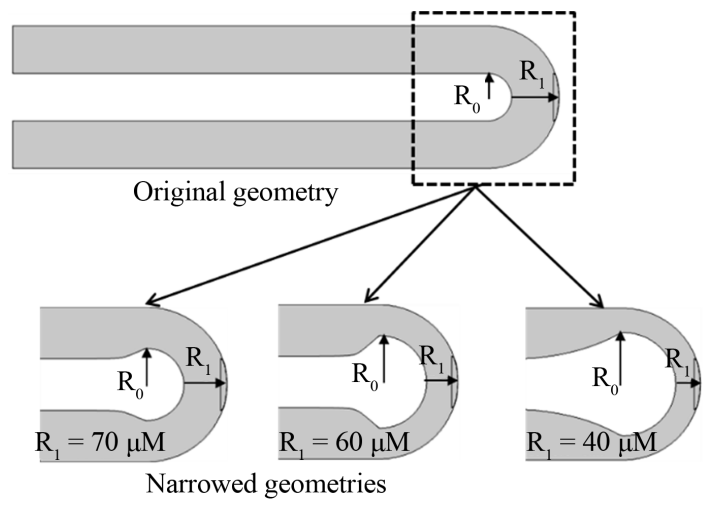

(a)

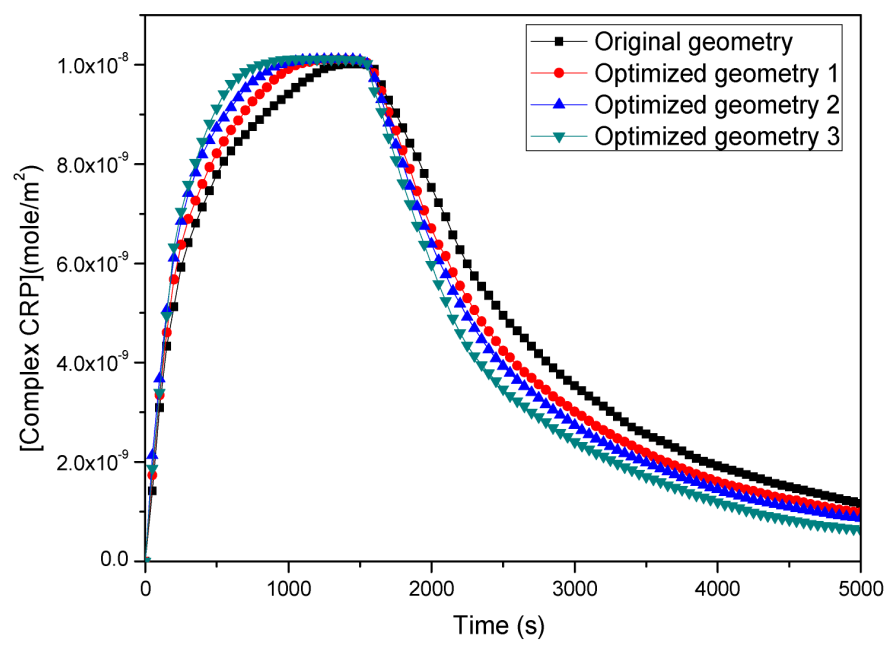

(b)

Figure 5. (a) Local narrowing of the bent zone following the two specific narrowing dimensions $\left(R_{0}\right)$ and $\left(R_{1}\right)$. (b) Temporal evolution of the CPR complex concentration using the original channel and the three optimized channels at a constant inlet flow velocity and bulk concentration of $U=100 \mu \mathrm{m} / \mathrm{s}$ and is $6.4 \mathrm{nM}$, respectively.

Table 1. Initial slope and enhancement factors of CRP binding reaction association and dissociation phases for different narrowing dimensions $\left(R_{0}\right)$ and $\left(R_{1}\right)$.

\begin{tabular}{ccccccc}
\hline Geometry & $\begin{array}{c}R_{1} \\
(\mu \mathrm{m})\end{array}$ & $\begin{array}{c}R_{0} \\
(\mu \mathrm{m})\end{array}$ & $\begin{array}{c}\text { Initial slope } \\
(\text { association) }\end{array}$ & $\begin{array}{c}\text { Initial slope } \\
(\text { dissociation) }\end{array}$ & $\begin{array}{c}\text { Enhancement } \\
\text { factor }^{*} \\
\text { (association) }\end{array}$ & $\begin{array}{c}\text { Enhancement } \\
\text { factor } \\
\text { (dissociation) }\end{array}$ \\
\hline $\begin{array}{c}\text { Original } \\
\text { geometry }\end{array}$ & 100 & 50 & $1.76 \times 10^{-11}$ & $-5.67 \times 10^{-12}$ & - & - \\
$\begin{array}{c}\text { Optimized } \\
\text { geometry 1 }\end{array}$ & 70 & 8 & $1.86 \times 10^{-11}$ & $-6.30 \times 10^{-12}$ & 1.05 & 1.11 \\
$\begin{array}{c}\text { Optimized } \\
\text { geometry 2 }\end{array}$ & 60 & 90 & $1.97 \times 10^{-11}$ & $-6.50 \times 10^{-12}$ & 1.12 & 1.15 \\
$\begin{array}{c}\text { Optimized } \\
\text { geometry 3 }\end{array}$ & 40 & 110 & $2.1 \times 10^{-11}$ & $-6.81 \times 10^{-12}$ & 1.19 & 1.20 \\
\hline
\end{tabular}

*The enhancement factors are defined as the ratio of the binding reaction slope with narrowing to that without narrowing. 
explained by the faster diffusion in transporting the fluid to reach the reaction surface at lower microchannel height. Thus, the decay of the diffusion boundary layer evolution is clarified by the CRP molecules confinement into a thin layer made by the height reduction overhead the reaction surface. Accordingly, a significant enhancement of the fluid velocity near the reaction surface, improvises the target biomolecule's consumption.

\section{Conclusion}

In this work, we present a 2D simulation of a CRP microfluidic biosensor based on a U-shaped channel using the finite element method. Decreasing the microchannel's equivalent height using local narrowing through simple channel engineering enhanced the CRP binding reaction's association and dissociation rates. Importantly, the lower height of the channel and the faster average inlet velocity have led to the reduction of the diffusion boundary layer and improved the biosensor's performance. This work can undoubtedly offer new insights for further investigation in microflow injection experimental studies.

\section{Conflicts of Interest}

The authors declare that they have no conflict of interest.

\section{Funding}

The authors received no direct funding for this research.

\section{Data Availability}

All data generated or analyzed during this study are included in this published article.

\section{References}

[1] Younes, T.M., AlKhedher, M.A., Shgier, K.A. and Al Taweel, F. (2019) U-Shape Acoustic Liquid Densitometer. Instrumentation Mesure Metrologie, 18, 123-128. https://doi.org/10.18280/i2m.180205

[2] Rechberger, A., Amsüss, R., Rossegger, S., Breidler, R. and Steiner, G. (2019) High Precision Vibration-Type Densitometers Based on Pulsed Excitation Measurements. Sensors, 19, Article No. 1627. https://doi.org/10.3390/s19071627

[3] Ong, K.S., Tong, W.L. and Choong, J.K. (2016) Performance of U-Tube Solar Water Heater with Vertical and Inclined Panels. International Journal of Low-Carbon Technologies, 11, 248-253. https://doi.org/10.1093/ijlct/ctt063

[4] Alsabbah, S. and Mughrabi, T. (2008) Neural Network-Based Waveguide Acoustic Gas Detector. Proceeding 5th International Symposium on Mechatronics and Its Applications (ISMA 2008), Amman, 27-29 May 2008, 1-4. https://doi.org/10.1109/ISMA.2008.4648867

[5] Younes, T.M., Alia, M.A.K. and Al-Sabbah, S. (2010) Acoustic Temperature Transducer. Sensors \& Transducers, 119, 46-57.

[6] Outcalt, S.L. and McLinden, M.O. (2007) Automated Densimeter for the Rapid 
Characterization of Industrial Fluids. Industrial \& Engineering Chemistry Research, 46, 8264-8269. https://doi.org/10.1021/ie070791e

[7] Fang, Y.-L., Wang, C.-T. and Chiang, C.-C. (2016) A Small U-Shaped Bending-Induced Interference Optical Fiber Sensor for the Measurement of Glucose Solutions. Sensors, 16, Article No. 1460. https://doi.org/10.3390/s16091460

[8] Raoufi, N., Surre, F., Rajarajan, M., Sun, T. and Grattan, K.T.V. (2014) Fiber Optic $\mathrm{pH}$ Sensor Using Optimized Layer-by-Layer Coating Approach. IEEE Sensors Journal, 14, 47-54. https://doi.org/10.1109/JSEN.2013.2280283

[9] Gao, S.S., Qiu, H.W., Zhang, C., Jiang, S.Z., Li, Z., Liu, X.Y., Yue, W.W., Yang, C., Huo, Y.Y., Feng, D.J. and Li, H.S. (2016) Absorbance Response of a Graphene Oxide Coated U-Bent Optical Fiber Sensor for Aqueous Ethanol Detection. RSC Advances, 6, 15808-15815. https://doi.org/10.1039/C5RA22211G

[10] Chiang, C.C. and Chao, J.C. (2013) Whispering Gallery Mode Based Optical Fiber Sensor for Measuring Concentration of Salt Solution. Journal of Nanomaterials, 2013, Article ID: 372625. https://doi.org/10.1155/2013/372625

[11] Llorente, V.B., Erro, E.M., Baruzzi, A.M. and Iglesias, R.A. (2015) Optochemical Fiber Sensor for Toluidine Blue Detection in High Turbidity Media. Sensors and Actuators B: Chemical, 216, 316-320. https://doi.org/10.1016/j.snb.2015.03.085

[12] Qiu, B., Gong, L., Li, Z. and Han, J. (2019) Electrokinetic Flow in the U-Shaped Micro-Nanochannels. Theoretical and Applied Mechanics Letters, 9, 36-42.

https://doi.org/10.1016/j.taml.2019.01.006

[13] Wen, H.Y., Huang, C.W., Le Li, Y., Chen, J.L., Yeh, Y.T. and Chiang, C.C. (2020) A Lamping U-Shaped Fiber Biosensor Detector for MicroRNA. Sensors, 20, Article No. 1509. https://doi.org/10.3390/s20051509

[14] Bharadwaj, R., Sai, V.V.R., Thakare, K., Dhawangale, A., Kundu, T., Titus, S., Verma, P.K. and Mukherji, S. (2011) Evanescent Wave Absorbance Based Fiber Optic Biosensor for Label-Free Detection of E. coli at $280 \mathrm{~nm}$ Wavelength. Biosensors and Bioelectronics, 26, 3367-3370. https://doi.org/10.1016/j.bios.2010.12.014

[15] Littlejohn, D., Lucas, D. and Han, L. (1999) Bent Silica Fiber Evanescent Absorption Sensors for Near-Infrared Spectroscopy. Applied Spectroscopy, 53, 845-849.

https://doi.org/10.1366/0003702991947423

[16] Radadia, A.D., Salehi-Khojin, A., Masel, R.I. and Shannon, M.A. (2010) The Effect of Microcolumn Geometry on the Performance of Micro-Gas Chromatography Columns for Chip Scale Gas Analyzers. Sensors and Actuators B: Chemical, 150, 456-464. https://doi.org/10.1016/j.snb.2010.07.002

[17] Molho, J.I., Herr, A.E., Mosier, B.P., Santiago, J.G., Kenny, T.W., Brennen, R.A., Gordon, G.B. and Mohammadi, B. (2001) Optimization of Turn Geometries for Microchip Electrophoresis. Analytical Chemistry, 73, 1350-1360.

https://doi.org/10.1021/ac001127+

[18] Griffiths, S.K. and Nilson, R.H. (2000) Band Spreading in Two-Dimensional Microchannel Turns for Electrokinetic Species Transport. Analytical Chemistry, 72, 5473-5482. https://doi.org/10.1021/ac000595g

[19] Paegel, B.M., Hutt, L.D., Simpson, P.C. and Mathies, R.A. (2000) Turn Geometry for Minimizing Band Broadening in Microfabricated Capillary Electrophoresis Channels. Analytical Chemistry, 72, 3030-3037. https://doi.org/10.1021/ac000054r

[20] Bahri, M., Dermoul, I., Getaye, M., Ben Ali, M. and Abdelhamid, E. (2019) 2D Simulation of a Microfluidic Biosensor for CRP Detection into a Rotating Micro-Channel. SN Applied Sciences, 1, Article No. 1199.

https://doi.org/10.1007/s42452-019-1231-8 
[21] Selmi, M., Echouchene, F., Gazzah, M.H. and Belmabrouk, H. (2015) Flow Confinement Enhancement of Heterogeneous Immunoassays in Microfluidics. IEEE Sensors Journal, 15, 7321-7328. https://doi.org/10.1109/ISEN.2015.2475610

[22] Sigurdson, M., Wang, D. and Meinhart, C.D. (2005) Electrothermal Stirring for Heterogeneous Immunoassays. Lab on a Chip, 5, 1366-1373.

https://doi.org/10.1039/b508224b

[23] Munir, A., Wang, J., Li, Z. and Zhou, H.S. (2010) Numerical Analysis of a Magnetic Nanoparticle-Enhanced Microfluidic Surface-Based Bioassay. Microfluidics and Nanofluidics, 8, 641-652. https://doi.org/10.1007/s10404-009-0497-3

[24] Selmi, M., Echouchene, F. and Belmabrouk, H. (2016) Analysis of Microfluidic Biosensor Efficiency Using a Cylindrical Obstacle. Sensor Letters, 14, 26-31. https://doi.org/10.1166/sl.2016.3527

[25] Selmi, M., Khemiri, R., Echouchene, F. and Belmabrouk, H. (2016) Electrothermal Effect on the Immunoassay in a Microchannel of a Biosensor with Asymmetrical Interdigitated Electrodes. Applied Thermal Engineering, 105, 77-84. https://doi.org/10.1016/j.applthermaleng.2016.05.132

[26] Azad, N., Rojanasakul, Y. and Vallyathan, V. (2008) Inflammation and Lung Cancer: Roles of Reactive Oxygen/Nitrogen Species. Journal of Toxicology and Environmental Health, Part B, 11, 1-15. https://doi.org/10.1080/10937400701436460

[27] Pepys, M.B. and Hirschfield, G.M. (2003) C-Reactive Protein: A Critical Update. Journal of Clinical Investigation, 111, 1805-1812. https://doi.org/10.1172/JCI200318921

[28] Ji, M., Du, L., Ma, Z., Xie, J., Huang, Y., Wei, X., Jiang, X., Xu, J., Yin, R., Wang, Y., Dai, J., Jin, G., Xu, L., Zhu, C., Hu, Z., Ma, H., Zhu, M. and Shen, H. (2022) Circulating C-Reactive Protein Increases Lung Cancer Risk: Results from a Prospective Cohort of UK Biobank. International Journal of Cancer, 150, 47-55. https://doi.org/10.1002/ijc.33780

[29] Yi, M., Zhang, H., Qi, S. and Li, Q. (2016) Determination of C-Reactive Protein Concentration in Serum Based on Chemiluminescence Analysis. 2016 8th International Conference on Measuring Technology and Mechatronics Automation (ICMTMA 2016), Macau (China), 11-12 March 2016, 214-217. https://doi.org/10.1109/ICMTMA.2016.61

[30] Kuzmin, D. and Hämalä̈nen, J. (2016) Finite Element Methods for Computational Fluid Dynamics : A Practical Guide. Society for Industrial and Applied Mathematics, Philadelphia.

[31] COMSOL Multiphysics ${ }^{\circledast}$ Modeling Software (n.d.). https://www.comsol.com/

[32] Yang, C.K., Chang, J.S., Chao, S.D. and Wu, K.C. (2008) Effects of Diffusion Boundary Layer on Reaction Kinetics of Immunoassay in a Biosensor. Journal of Applied Physics, 103, Article ID: 084702. https://doi.org/10.1063/1.2909980

[33] Thompson, J.A. and Bau, H.H. (2010) Microfluidic, Bead-Based Assay: Theory and Experiments. Journal of Chromatography B, 878, 228-236.

https://doi.org/10.1016/j.jchromb.2009.08.050

[34] Huang, K.R. and Chang, J.S. (2013) Three Dimensional Simulation on Binding Efficiency of Immunoassay for a Biosensor with Applying Electrothermal Effect. Heat and Mass Transfer, 49, 1647-1658. https://doi.org/10.1007/s00231-013-1214-Z

[35] Sadana, A. and Vo-Dinh, T. (1997) Antibody-Antigen Binding Kinetics a Model for Multivalency Antibodies for Large Antigen Systems. Biotechnology and Applied Biochemistry, 67, 1-22. https://doi.org/10.1007/BF02787837 
[36] Zimmermann, M., Delamarche, E., Wolf, M. and Hunziker, P. (2005) Modeling and Optimization of High-Sensitivity, Low-Volume Microfluidic-Based Surface Immunoassays. Biomedical Microdevices, 7, 99-110.

https://doi.org/10.1007/s10544-005-1587-y

[37] Stone, H.A., Stroock, A.D. and Ajdari, A. (2004) Engineering Flows in Small Devices: Microfluidics toward a Lab-on-a-Chip. Annual Review of Fluid Mechanics, 36, 381-411. https://doi.org/10.1146/annurev.fluid.36.050802.122124

[38] Aoun, N., Echouchene, F., Diallo, A.K., Launay, J. and Belmabrouk, H. (2016) Finite-Element Simulations of the $\mathrm{pH}-$ ElecFET Microsensors. IEEE Sensors Journal, 16, 6519-6526. https://doi.org/10.1109/JSEN.2016.2585506

[39] Chaiken, I., Rosé, S. and Karlsson, R. (1992) Analysis of Macromolecular Interactions Using Immobilized Ligands. Analytical Biochemistry, 201, 197-210. https://doi.org/10.1016/0003-2697(92)90329-6

[40] Levich, V. (1962) Physicochemical Hydrodynamics. Englewood Cliffs, Prentice-Hall. http://www.worldcat.org/title/physicochemical-hydrodynamics/oclc/1378432

[41] Hofmann, O., Voirin, G., Niedermann, P. and Manz, A. (2002) Three-Dimensional Microfluidic Confinement for Efficient Sample Delivery to Biosensor Surfaces. Application to Immunoassays on Planar Optical Waveguides. Analytical Chemistry, 74, 5243-5250. https://doi.org/10.1021/ac025777k 